\title{
Application of microscope thermography in the production technology of semiconductor lasers
}

\author{
M. Bednarek*, J. Rybiński*, T. Świetlik**, P. Wiśniewski**
}

\author{
* The Main School of Fire Service \\ ** Institute of High Pressure Physics of the Polish Academy of Sciences
}

\section{Abstract}

A thermal camera with a microscope attachment was applied to appraise the effectiveness of heat dissipation generated within the epitaxial layers of a laser diode.

Tests were conducted on GaN-based, blue light emitting devices mounted by means of two alternative packaging schemes. The tests were aimed at finding the most effective way of heat dissipation, resulting in reduced temperature of the device's active region, which has a beneficial influence on a laser performance.

\section{Subject of tests}

The subject of the tests were blue semiconductor lasers built on the basis of gallium nitride and its ternary compounds (InGaN, AIGaN). Continuous duty semiconductor lasers are fittings exposed to release of considerable heat quantities in the active area with a very low volume. This leads to temperature growth in the active area which significantly worsens the laser parameters, and may even prevent a given device from lasing. Consequently effective heat dissipation becomes a crucial issue, both in terms of achieving high output power at continuous wave working regime and also prolonged lifetime of the laser.

The conducted experiments were aimed at checking temperature distribution in the semiconductor laser assembled within a laser casing $5.6 \mathrm{~mm}$ with a diamond heat distribution separator plate, to allow appraising the effectiveness of heat abstraction from its active area. This concerned in particular detecting places with the highest thermal resistance in the structure: laser active structure (laser strip) - weld (AuSn) - diamond plate weld (AuSn) - casing 5.6mm (SPC - silver plater copper) - radiator.

\section{Measurement stand}

A Raytheon Radiance HSX camera was used to record temperature distribution. This is a radiometric camera with cooled InSb detector, which operates within the spectral range of 3-5 $\mu \mathrm{m}$. Its exceptional versatility arises from unlimited access of the user to its elementary parameters: detector integration time, detector operation mode, frame frequency. The detector integration time is set within the range of $0.002-14 \mathrm{~ms}$ and allows optimisation of camera settings for the specific temperature range, required in the given experiment. Such optimisation allows achieving a high temperature resolution of $\mathrm{NEDT}=0.025^{\circ} \mathrm{C}$. The Raytheon microscope attachment assures maximum spatial resolving power of $8 \mu \mathrm{m}$. The required minimum distance of the specimen from the attachment lens equals to $33 \mathrm{~mm}$.

The main difficulty arises from determining the surface emission coefficient. Coefficients provided in the literature do not take the surface microstructure into consideration. Improper attribution of the coefficient value would cause false temperature readings of the examined objects. This problem can be solved through camera calibration that employs the examined item as a standard. The assigned temperature was achieved with the use of the Peltier Module and controlled by the appropriately assembled thermocouple.

The Radiance HSX camera is equipped with a photon detector. During the detector reading phase the current amperage is proportional to the charge collected in the FPA element that in turn corresponds to the number of the photons absorbed during the irradiation. The analog signal is transformed by the 12-bit A/D converter. Digital signal reaches the computer through a frame grabber and can be interpreted as the radiation strength reading. The 12-bit converter allows dividing the camera dynamics (that depends on adjustable detector integration time) into 4,096 levels. The calibration procedure consists in ascribing the temperature values corresponding to signal values, and adjusting the calibration curve to these value pairs. Applying emission coefficient approaching one, the gained calibration requires introduction of the emission coefficient of the examined object. It is measured with the thermographic programme equipment. Such correction is not necessary when the surveyed object is employed as a template. This, however, requires maintaining identical conditions of calibration and further measurements, on account of the influence of background emission, atmosphere, etc. These conditions were monitored with the second thermographic InfraCAM camera. Using this camera during observation of the whole stand allows for elimination of the additional emission sources, namely the camera operator or other research participants. Should they change the position, they may influence the research environment. Figure 1 demonstrates the representation of the calibration curves. 


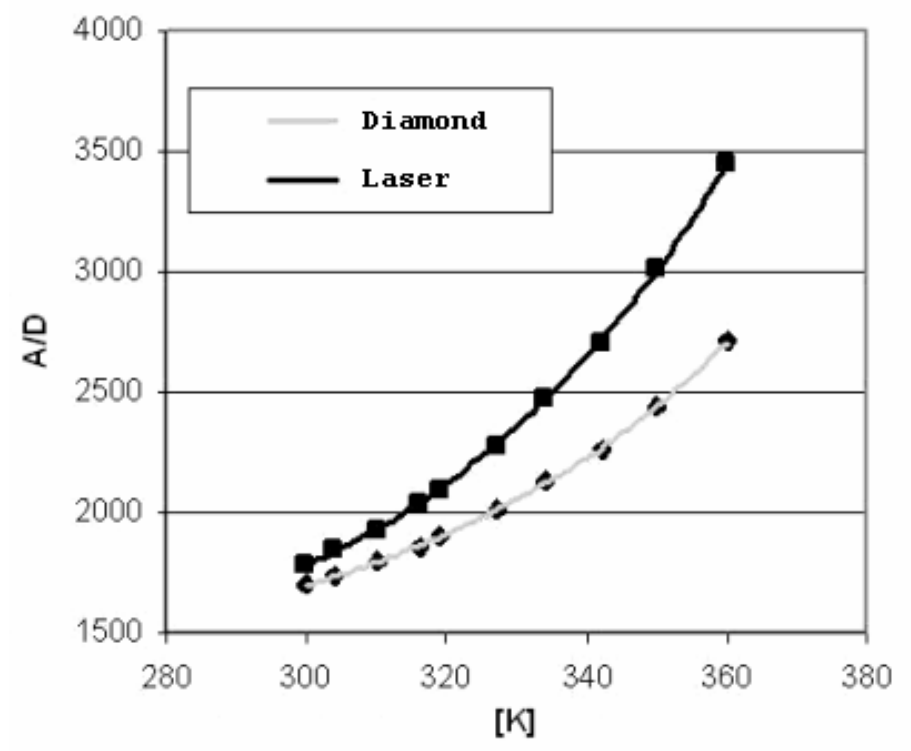

Fig.1. Calibration curves.

During the microscopic measurements appears another difficulty connected with spatial calibration of the detector (NUC). The optical system of the camera does not guarantee a uniform irradiation of the FPA. The camera detector technology results in minor but noticeable parameter differences between single photosensitive points. In the Radiance HSX camera, the detector signal is read in two independent tracks. One of them supports even, and the other - odd lines of the picture. This process takes place in the analog part of the camera hence it is impossible to provide a maximum symmetry of the tracks. All the enumerated imperfections may be adjusted to a target value with the table of 65,536 gain and offset coefficients for all detector pixels. This type of calibration requires emission templates that have normalised temperature and emission coefficient for the entire surface.

Microscopic measurements with micrometer spatial resolution require considerable stand stability. Any vibrations of the camera, optical system or the object that have amplitude comparable to the spatial resolution disqualify the measurements. Although it is possible to correct the vibrations and track the assigned point trajectory in the thermographic scene, the difficulty is not insignificant and requires separate study. Such problems occur during observations conducted by helicopter or plane. Gyroscope and pneumatic systems do not guarantee stability obliged in microscopic measurements.

During the construction of the measurement stand for the microscope thermographic investigation it was intended to provide maximum system stability through using the optical bench base along with the optical rail and the system of stands of appropriate weight. All pieces of equipment and the examined object were stiffly aligned in order to maintain phase and amplitude coherence. Thus vibration does not influence the stability of thermogram sequences.

\section{Experiment}

The laser was assembled on a diamond heat spreader plate with the active area towards the diamond using Au0.8Sn0.2 eutectic mixture. The diamond plate was assembled on a standard copper laser submount placed in a $5.6 \mathrm{~mm}$ brass grip with the use of Au0.8Sn0.2 eutectic mixture.

Layers type $p$ and socket for type $p$ were the main heat source in the structure. Electric power used during laser operation equalled to ca. $3 \mathrm{~W}$. It was necessary to find an effective way of heat abstraction. Otherwise the emitted heat would lead to a temperature increase, and consequently: raising threshold current, reducing the range of external temperatures which allow lasing and quicker degradation of the element.

To improve temperature stability and to reduce temperature in the active area a copper block was additionally introduced, which covers the laser diode on top (Fig. 3). This led to a significant temperature reduction in the active area: from $87^{\circ} \mathrm{C}$ (point $\mathrm{A}$ on Fig.2) to $47^{\circ} \mathrm{C}$ (point $\mathrm{F}$ on Fig. 3). Less heat remains to be abstracted through the diamond. As a consequence heat is not being accumulated on the point of contact of diamond-copper (no temperature gradient occurs on the thermogram). 


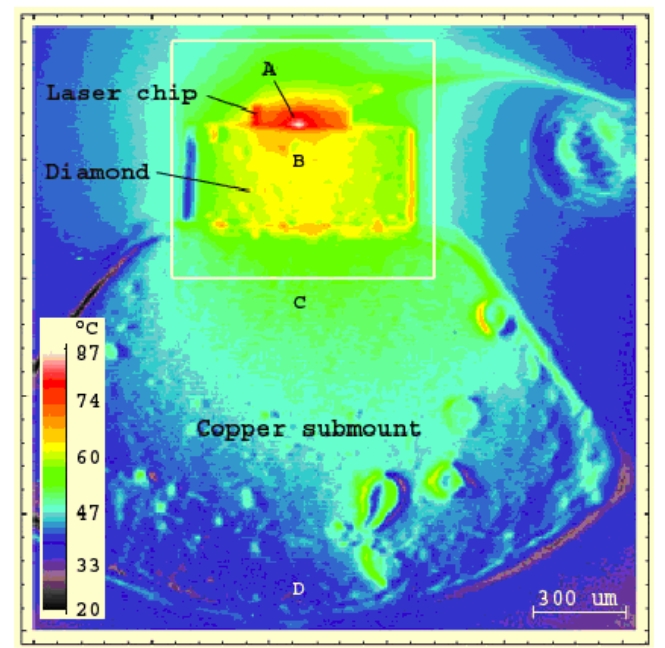

Fig.2. Thermogram of laser element surface - temperature of active area emitting the laser beam (point $A)$ equals to ca. $87^{\circ} \mathrm{C}$.

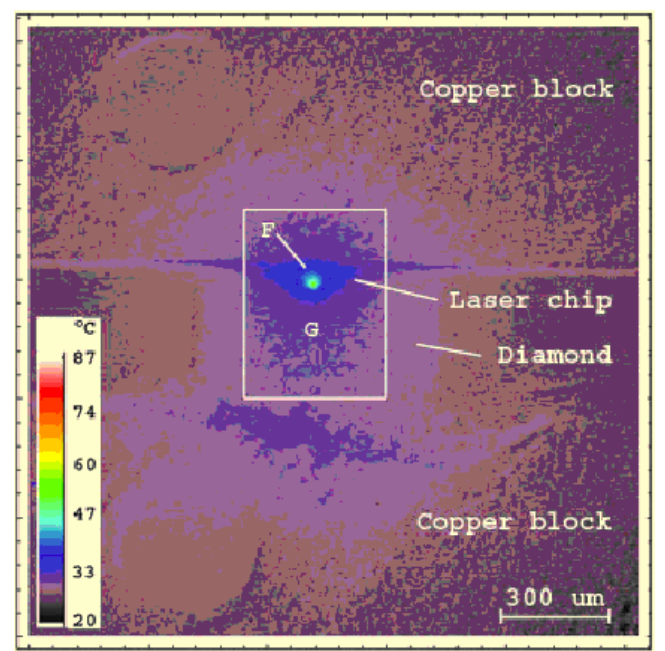

Fig. 3. Thermogram of laser element surface with additional copper block which covers the top of the laser diode - temperature of active area which emits the laser beam (point $F$ ) equals to ca. $47^{\circ} \mathrm{C}$.

In both cases the laser was stabilised with respect to temperature on a peltier thermoelectric stabiliser. The base temperature was maintained on a constant level of $18^{\circ} \mathrm{C}$.

A comparison of evolution taking place in temperature on the active area shows supremacy of assembly with a double copper block over assembly without additional heat abstraction on top. A laser in a copper casing heats up to a much smaller extent. It heats up much less, and becomes cooler much quicker following completion of impulse. No temperature fluctuations occur as in the event of a standard casing. The laser works in a stable way without the effect of structure overheating.

\section{Conclusions}

The applied assembly method with a double copper block seems to be a promising technique, which guarantees a satisfactory effectiveness of heat dissipation. By lowering overall temperature, the laser action may be achieved at much lower supply current which guarantees prolonged longevity of laser diodes.

This could be proved thanks to the application of the microscope thermography method. 
http://dx.doi.org/10.21611/qirt.2008.10_01_07 